\title{
NUEVAS LOCALIDADES EN LA DISTRIBUCIÓN DE MURCIÉLAGOS FILOSTÓMINOS (CHIROPTERA: PHYLLOSTOMIDAE) EN CHIAPAS, MÉXICO
}

\section{LUIS ARTURO HERNÁNDEZ-MIJANGOS ${ }^{1}$, ROBERTO GÁLVEZ MEJÍA², MARIANNA DÍAZ NEGRETE ${ }^{1}$ Y CARLOS MAURICIO CRUZ DURANTE $^{1}$}

\author{
${ }^{1}$ Dirección de Áreas Naturales y Vida Silvestre, Secretaría de Medio Ambiente y Vivienda, \\ Av. Río Usumacinta No. 851, Col. Los Laguitos, C. P. 29020, \\ Tuxtla Gutiérrez, Chiapas, México. \\ ${ }^{2}$ Instituto Internacional en Conservación y Manejo de Vida Silvestre, Universidad Nacional, \\ Apdo. 1350-3000, Heredia, Costa Rica. \\ correo electrónico: stenops06@yahoo.com.mx
}

\begin{abstract}
We captured bats in natural protected areas in Chiapas, Mexico, including five individuals of the species Macrophyllum macrophyllum, Micronycteris schmidtorum, and Vampyrum spectrum. We report new localities on the distribution of this three species, extending their distribution ranges.
\end{abstract}

KEY WORDS: Chiapas, distribution, Macrophyllum macrophyllum, Micronycteris schmidtorum, Vampyrum spectrum.

PALABRAS CLAVE: Chiapas, distribución, Macrophyllum macrophyllum, Micronycteris schmidtorum, Vampyrum spectrum.

En México, la familia Phyllostomidae es la que comprende el mayor número de especies de quirópteros catalogadas en alguna categoría de riesgo, dentro de la cual la mayor proporción de especies corresponden a la subfamilia Phyllostominae (SEMARNAT, 2002). Los murciélagos filostóminos son especialmente sensibles a la perturbación antropogénica, debido a la dieta especializada y el uso de refugios con características muy específicas (Medellín et al., 2000); entre los cuales se encuentran Macrophyllum macrophyllum, Micronycteris schmidtorum y Vampyrum spectrum. Macrophyllum macrophyllum se distribuye desde Tabasco hasta Sudamérica (Hall, 1981), teniendo en México su distribución marginal. La distribución de $M$. schmidtorum abarca desde la península de Yucatán y Chiapas hasta Sudamérica (Escobedo-Cabrera et al., 2006); mientras que la distribución de $V$. spectrum abarca desde Veracruz en México hasta Sudamérica (Navarro y Wilson, 1982). En México, las tres especies han sido registradas principalmente en áreas de bosque tropical perennifolio (Navarro, 1979; ÁlvarezCastañeda y Álvarez, 1991; López et al., 1998) y están clasificadas en categorías de riesgo de extinción (SEMARNAT, 2002). El presente documento tiene la finalidad de 
aportar nuevas localidades y extensión de la distribución conocida para $M$. macrophyllum, M. schmidtorum y V. spectrum.

Como parte de las actividades del proyecto "Monitoreo Biológico en Áreas Naturales Protegidas", ejecutado por el Instituto de Historia Natural del estado de Chiapas, llevamos a cabo sesiones de captura de murciélagos en 12 localidades de Chiapas, entre febrero de 2005 y diciembre de 2007. Las capturas se llevaron a cabo disponiendo cinco redes de niebla de $12 \mathrm{~m}$ de longitud y $3 \mathrm{~m}$ de ancho, entre la vegetación al nivel del sotobosque. Durante estos muestreos registramos a $M$. macrophyllum, M. schmidtorum y V. spectrum en cuatro nuevas localidades.

\section{Macrophyllum macrophyllum (Schinz, 1821)}

El 30 de octubre de 2006 capturamos una hembra juvenil (medidas somáticas: 70.8-3512.7-14.7 $\equiv 7 \mathrm{AB}=34.5$; medidas craneales: $\mathrm{LCC}=11.4, \mathrm{LCB}=13.35, \mathrm{LDM}=5.32$, $\mathrm{AC}=7.18$, $\mathrm{AI}=3.82$ ) en la Reserva de la Biosfera La Encrucijada (1504’03" N, 924' $18^{\circ}$ " W, 10 msnm). El espécimen fue capturado junto con Artibeus jamaicensis y Desmodus rotundus. La captura se realizó con una red de niebla dispuesta al nivel del sotobosque, en una asociación de manglar, selva mediana subcaducifolia y palmar. En el sitio de colecta están presentes especies arbóreas como Rhizophora mangle, Laguncularia racemosa, Bursera simaruba, Guazuma ulmifolia, Cynometra oaxacana, Sabal mexicana y Acrocomia mexicana. Las localidades de colecta conocidas con anterioridad para la especie en México se ubican en Tabasco y en la Selva Lacandona en Chiapas (Lay, 1963; Álvarez-Castañeda y Álvarez, 1991), por lo que este registro es el primero proveniente de la vertiente del Pacífico mexicano (Hernández-Mijangos et al., 2008). Nuestro registro extiende la distribución de la especie 234 km en dirección suroeste respecto a los registros de la Selva Lacandona (Figura 1). En México parece ser una especie rara, considerando los pocos individuos colectados (menos de cinco; R. Medellín, com. pers.). El espécimen está depositado en la Colección Zoológica Regional (Mammalia) del Instituto de Historia Natural (CZRMA), con número de catálogo 2575.

\section{Micronycteris schmidtorum Sanborn, 1935}

Obtuvimos tres individuos en diferentes localidades de Chiapas. El primer individuo fue un macho adulto (medidas somáticas: 55.5-11.49-10.1-21.7 $\equiv$ ? $\mathrm{AB}=35.1$; medidas craneales: LCC=19.94, LCB=18.21, LDM=7.9, AC=?, AI=4.32; CZRMA-2533), capturado el 23 de agosto de 2005, en la ranchería Las Mandarinas a $15 \mathrm{~km}$ al norte de Mapastepec (15³1’41" N, 9253’15" W, 235 msnm). La vegetación predominante en el sitio es de selva mediana subperennifolia, dominada por Brosimum alicastrum, Terminalia oblonga, Cupania dentata, Aspidosperma cruentum y Aphananthe 


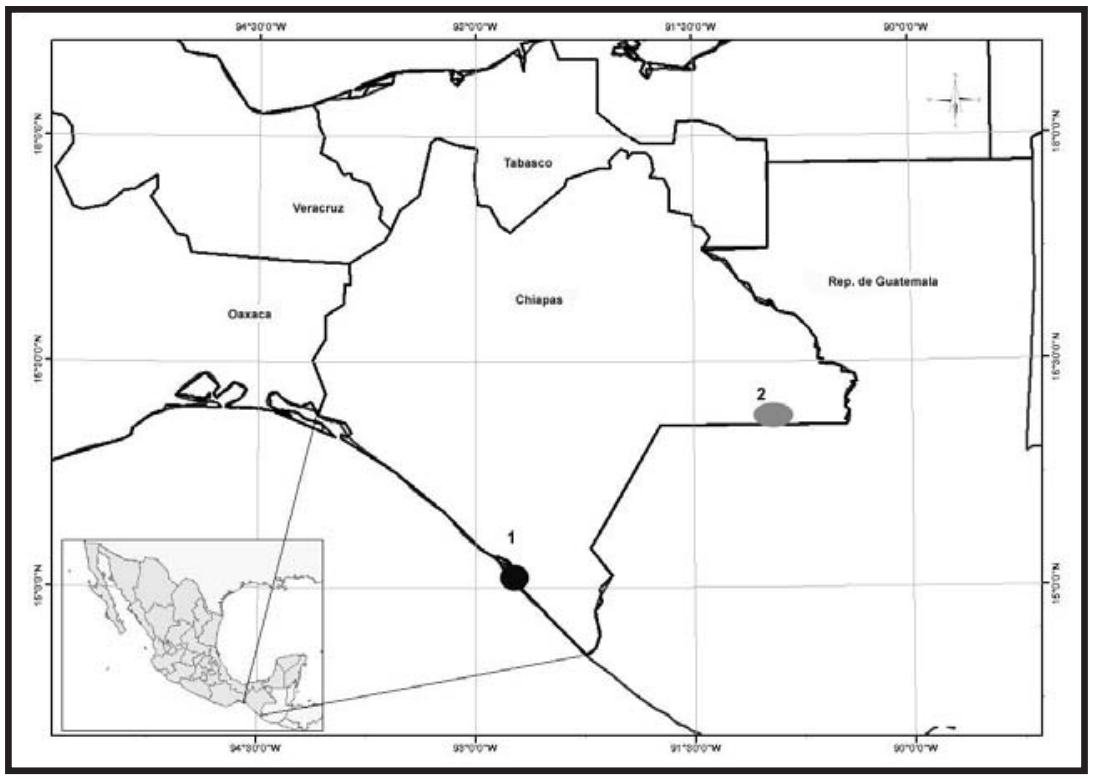

Figura 1. Registros de Macrophyllum macrophyllum en Chiapas, México. 1: Nuevo registro y 2: Registro anterior.

monoica; y perturbada en su estrato herbáceo y arbustivo. En México, la distribución histórica de $M$. schmidtorum está restringida a ocho localidades de los estados de Yucatán, Quintana Roo, Campeche y Chiapas (Jones et al., 1973; Álvarez-Castañeda y Álvarez, 1991; Vargas-Contreras et al., 2004; Escobedo-Cabrera et al., 2006). En Chiapas únicamente se le había registrado en Yaxchilán, en la Selva Lacandona (Álvarez y Álvarez-Castañeda, 1990). En México, este es el primer registro de la especie proveniente de la vertiente del Pacífico (Hernández-Mijangos et al., 2008). Nuestro registro se localiza a 255 km de Yaxchilán en dirección suroeste (Figura 2).

El segundo individuo fue capturado el 27 de octubre de 2005 y corresponde a un macho adulto (medidas somáticas: 62.5-12.73-9.7-17.78 $\equiv$ ? $\mathrm{AB}=35.52$; medidas craneales: $\mathrm{LCC}=20.17, \mathrm{LCB}=18.22, \mathrm{LDM}=8.02, \mathrm{AI}=4.23$; $\mathrm{CZRM}-2534)$. La captura se realizó en la Reserva de la Biosfera Selva El Ocote (1702’14" N, 9348’31" W, 275 msnm), confirmando de esta manera la hipótesis de la distribución de la especie en la parte norte de Chiapas (Álvarez-Castañeda y Álvarez, 1991). El sitio es cárstico, con vegetación de selva mediana subperennifolia en buen estado de conservación y presencia de especies arbóreas como Zanthoxylum procerum, Eupatorium pittieri, Stemmadenia donell-smithii, Xylosma flexuosa, Sapindus saponaria, Luehea candida, Lonchocarpus schiedeanus y B. simaruba. Este sitio de colecta se encuentra a 
305 km de Yaxchilán en dirección oeste; respecto al registro de Mapastepec, la distancia es de 195 km en dirección nor-noroeste (Figura 2).

El tercer individuo correspondió a una hembra adulta (medidas somáticas: 58.6-11.4-9.5-17.9 $\equiv$ ?, $\mathrm{AB}=35$; medidas craneales: $\mathrm{LCC}=35.8, \mathrm{LCB}=44.2, \mathrm{LDM}=20.52$, $\mathrm{AC}=23.76, \mathrm{AI}=10.32$ ), sin signos reproductivos y fue capturada en el ejido "3 de Mayo", 20 km al norte de Mapastepec (15³4’12"N,9251’37"W; Gálvez, 2008). Este registro se localiza $5 \mathrm{~km}$ al norte del registro antes mencionado (Figura 2), dentro de la zona de amortiguamiento de la Reserva de la Biosfera El Triunfo. La captura se realizó en septiembre de 2006, en un cafetal con sombra de árboles de las especies $B$. alicastrum, A. cruentum, Cymbopetalum penduliflorum, Trema micrantha, T. oblonga, L. guatemalensis y Erythroxylum tabascense. Al momento de la captura, en la misma red se encontraron individuos de A. jamaicensis y Sturnira lilium. Micronycteris schmidtorum es una especie considerada rara, debido a los pocos individuos colectados.

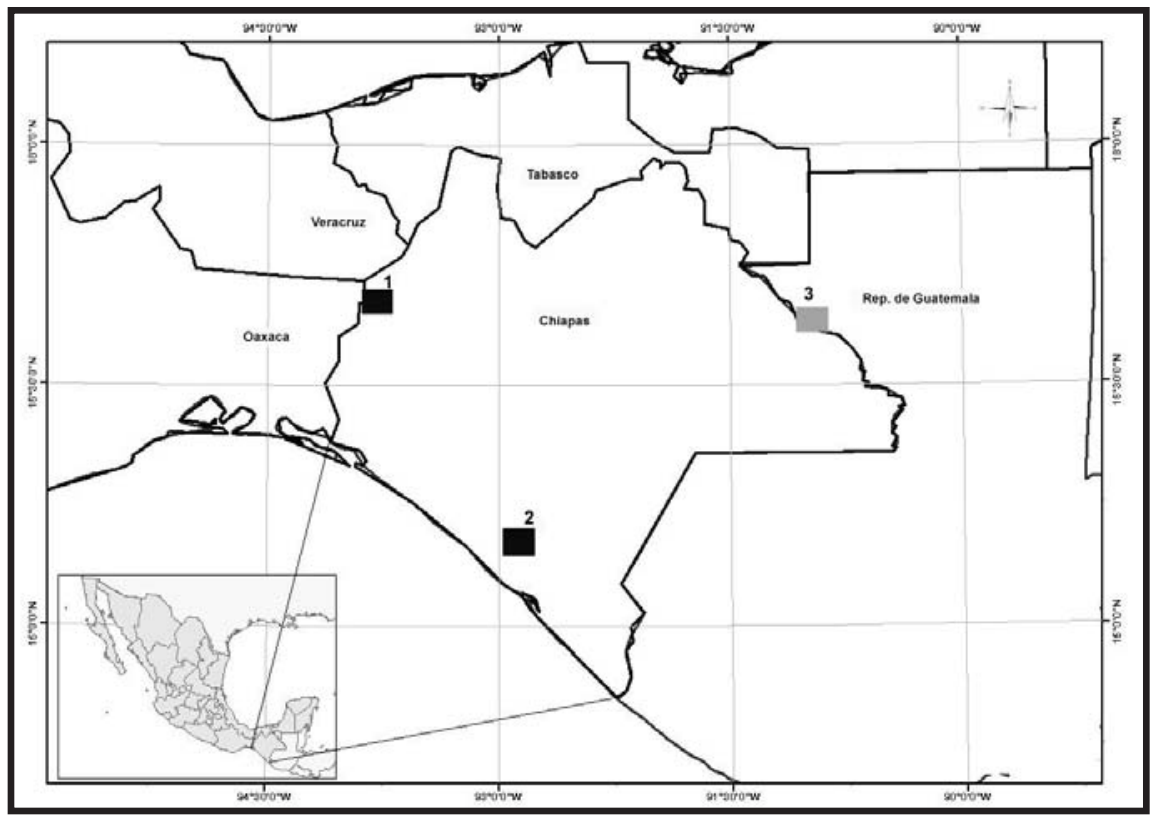

Figura 2. Registros de Micronycteris schmidtorum en Chiapas, México. 1: Selva El Ocote; 2: Mapastepec (dos registros nuevos) y 3: Registro anterior. 


\section{Vampyrum spectrum (Linnaeus, 1758)}

El 9 de abril de 2007 capturamos una hembra adulta, reproductivamente inactiva (medidas somáticas: $135-0-29-41 \equiv 96 \mathrm{AB}=109$; medidas craneales: $\mathrm{LCC}=35.8, \mathrm{LCB}=$ 44.2, $\mathrm{LDM}=20.52, \mathrm{AC}=23.76, \mathrm{AI}=10.32$; CZRMA-2574) en la Reserva de la Biosfera Selva El Ocote (1702’14" N, 9348’31" W, 275 msnm). La captura se realizó mediante una red de niebla colocada entre la vegetación, al nivel del sotobosque. Esta es la segunda localidad de colecta de la especie en Chiapas, pues anteriormente era conocida sólo de la Selva Lacandona (López et al., 1998). Nuestro registro se localiza 325 km al oeste de la localidad de la Selva Lacandona (Figura 3). El sitio de colecta se ubica en una zona cárstica, con vegetación de selva mediana subperennifolia, dominada por especies arbóreas como Z. procerum, E. pittieri, S. donell-smithii, X. flexuosa, S. saponaria, L. candida, L. schiedeanus y B. simaruba. En México ha sido registrada en los estados de Campeche, Chiapas y Veracruz (Navarro, 1979; Lopez et al., 1998; Hernández-Huerta et al., 2000; Escalona-Segura et al., 2002). Nuestro registro confirma la distribución potencial de la especie en Chiapas (Hernández-Mijangos et al., 2008), de la que se especulaba que abarcaba las selvas de la zona norte del Estado que se conectan con Veracruz (Medellín et al., 1997). Esta especie es rara en México.

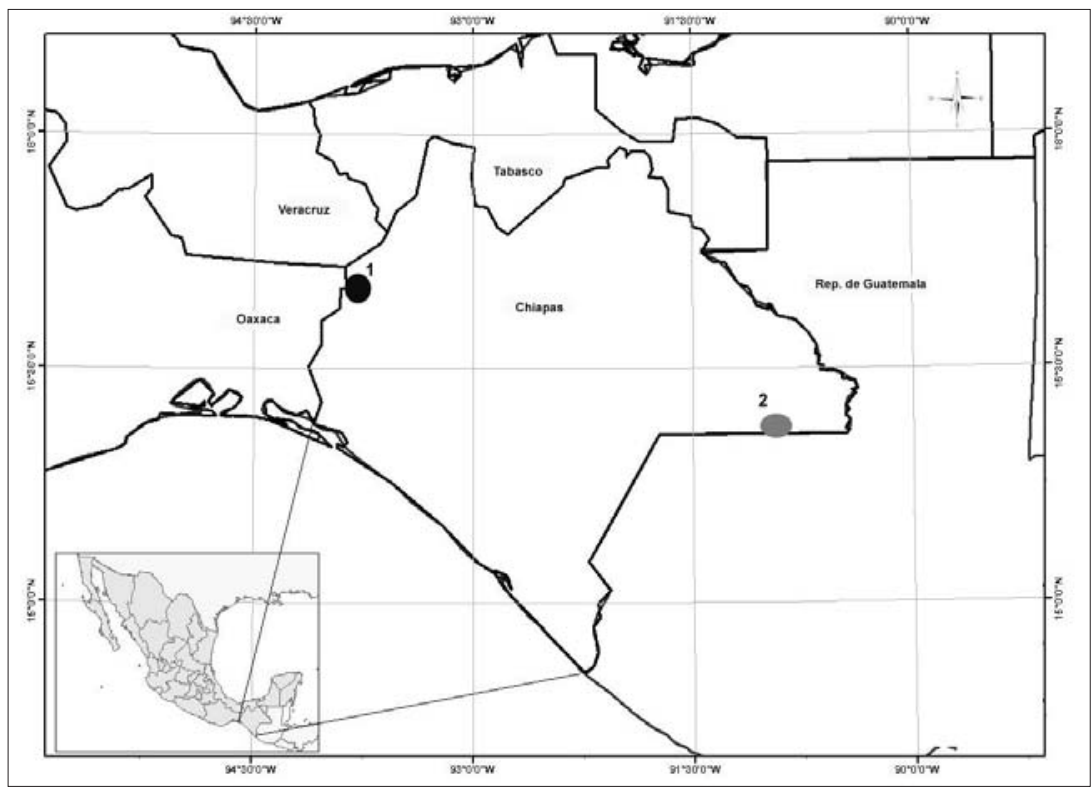

Figura 3. Registros de Vampyrum spectrum en Chiapas, México. 1: Nuevo registro, 2: Registro anterior. 
Macrophyllum macrophyllum, $M$. schmidtorum y V. spectrum son especies con bajos números de captura y de distribución restringida en México. Estas características, aunadas a los requerimientos de hábitat tan específicos que presentan, han originado su inclusión y permanencia como especies protegidas por la NOM-059ECOL-2001 (SEMARNAT, 2002), debido a los riesgos de extinción que enfrentan. Sin embargo, estudios que revelen la ampliación de ámbitos de distribución, o bien aporten datos que contribuyan al mejor conocimiento de sus tamaños poblacionales o de nuevos hábitat de distribución de estas y otras especies con problemáticas similares, son fundamentales para dilucidar su real situación de riesgo y establecer acciones que favorezcan su conservación.

\section{AGRADECIMIENTOS}

Agradecemos al gobierno del estado de Chiapas por el financiamiento del proyecto "Monitoreo Biológico en Áreas Naturales Protegidas" y a la Comisión Nacional de Áreas Naturales Protegidas por el apoyo otorgado. Asimismo, extendemos nuestro agradecimiento al Dr. Juan Carlos López Vidal por su apoyo en la identificación de los ejemplares de M. schmidtorum. Al Dr. Rodrigo Medellín, al Dr. Jorge Vargas y a un revisor anónimo por las sugerencias vertidas para el mejoramiento del manuscrito. De igual manera, agradecemos a Saúl Hernández por la elaboración de los mapas, así como a Marco Tulio Argueta, Rafael Pérez, Kaleb Zárate y al personal del proyecto mencionado, por su apoyo en las actividades de campo.

\section{LITERATURACITADA}

Álvarez-Castañeda, S., T. y T. Álvarez. 1991. Los Murciélagos de Chiapas. Instituto Politécnico Nacional. México.

Álvarez, T. y S.T. Álvarez-Castañeda. 1990. Cuatro nuevos registros de murciélagos (Chiroptera) del estado de Chiapas, México. Anales de la Escuela Nacional de Ciencias Biológicas, México, 33:157-161.

Escalona-Segura, G., J.A. Vargas-Contreras y L. Interián-Sosa. 2002. Registros importantes de mamíferos para Campeche, México. Revista Mexicana de Mastozoología, 6:166-170.

Escobedo-Cabrera, E., L. León-Paniagua y J. Arroyo-Cabrales. 2006. Geographic distribution and some taxonomic comments of Micronycteris schmidtorum Sanborn (Chiroptera: Phyllostomidae) in Mexico. Caribbean Journal of Science, 42:129-135.

Gálvez, R. 2008. Influencia de la heterogeneidad espacial y temporal sobre la diversidad alfa, beta y gamma de especies de murciélagos, en el paisaje tropical de la Reserva El Triunfo, Chiapas, México. Tesis de Maestría. Universidad Nacional, Heredia, Costa Rica.

Hall, E.R. 1981. The mammals of North America. John Wiley \& Sons. New York.

Hernández-Huerta, A., V.J. Sosa, M. Aranda y J. Bello. 2000. Records of small mammals in the Calakmul Biosphere Reserve, Yucatan Peninsula. The Southwestern Naturalist, 45:340344. 
Hernández-Mijangos, L.A., E. Pineda, M. Díaz y R. Pérez. 2008. Adición de registros mastofaunísticos en Reservas de Biosfera de Chiapas, México. Vertebrata Mexicana, 21:19-31.

Jones, J.K. Jr., J.D. Smith y H.H. Genoways. 1973. Annotated checklist of mammals of the Yucatan peninsula, Mexico. Occasional Papers, Museum of Texas Tech University, 13:131.

Lay, D. 1963. Seis mamíferos nuevos para la fauna de México. Anales del Instituto de Biología, 33:373-377.

López, M.C., R.A. Medellín y G. Yanes. 1998. Vampyrum spectrum en Chiapas, México. Revista Mexicana de Mastozoología, 3:135-136.

Medellín, R., A., H. Arita y O. Sánchez. 1997. Identificación de los Murciélagos de México, Clave de Campo. Publicaciones Especiales No. 2, Asociación Mexicana de Mastozoología, A.C., México.

Medellín, R.A., M. Equihua y M. Amín. 2000. Bat diversity and abundance as indicators of disturbance in Neotropical rainforest. Conservation Biology, 14:1666-1675.

Navarro, D. 1979. Vampyrum spectrum (Chiroptera: Phyllostomatidae) in Mexico. Journal of Mammalogy, 60:435.

Navarro, D. y D.E. Wilson. 1982. Vampyrum spectrum. Mammalian Species, 184:1-4.

SEMARNAT. 2002. Norma Oficial Mexicana NOM-059-ECOL-2001, Protección ambientalEspecies nativas de México de flora y fauna silvestres-Categorías de riesgo y especificaciones para su inclusión, exclusión o cambio-Lista de especies en riesgo. Diario Oficial de la Federación, 6 de marzo de 2002, 1-56.

Vargas-Contreras, J.A., J.R. Herrera-Herrera y J.E. Escobedo-Cabrera. 2004. Noteworthy records of mammal from Campeche, Mexico. Revista Mexicana de Mastozoología, 8:61-69. 